\title{
INCONTINÊNCIA FECAL EM MULHERES NA PÓS-MENOPAUSA: prevalência, intensidade e fatores associados
}

\author{
Simone Caetano Morale de OLIVEIRA ${ }^{1}$, Aarão Mendes PINTO-NETO ${ }^{1}$, \\ Délio Marques CONDE ${ }^{1}$, Juvenal Ricardo Navarro GÓES ${ }^{2}$, \\ Danielle SANTOS-SÁ ${ }^{1}$ e Lúcia COSTA-PAIVA ${ }^{1}$
}

RESUMO - Racional - A incontinência fecal é mais freqüente na população feminina, tornando-se mais prevalente com o aumento da idade. Poucos estudos avaliaram sua prevalência e intensidade em mulheres na pós-menopausa. Objetivos Investigar a prevalência e os fatores associados à incontinência fecal em mulheres na pós-menopausa e estudar a intensidade dos sintomas. Métodos - Realizou-se estudo de corte transversal com 100 mulheres na pós-menopausa e idade superior a 45 anos. Foram avaliadas as características sociodemográficas e clínicas com análise descritiva das mesmas. Estimou-se a prevalência de incontinência fecal. Aplicou-se o escore de St. Mark para estudar a intensidade dos sintomas associados à incontinência fecal. A seguir, o escore foi categorizado de acordo com o tercil e a intensidade dos sintomas foi classificada em graus leve, moderado ou grave. Análises bivariada e multivariada foram utilizadas para estudar a associação entre a incontinência fecal e seus possíveis determinantes, empregando-se a razão de prevalência. $\mathrm{O}$ intervalo de confiança foi fixado em 95\%. Resultados - A média de idade foi de 58,9 \pm 5,9 anos (variação: 46-76 anos). A prevalência de incontinência fecal foi de 15\%. Das pacientes incontinentes, $60 \%$ apresentaram incontinência leve. Após análise multivariada, observouse como fator associado à incontinência fecal o antecedente de partos fórcipes (razão de prevalência: 7,80; intervalo de confiança 95\%: 2,38-25,55). Conclusões - A prevalência de incontinência fecal em mulheres na pós-menopausa foi elevada. Os dados sugerem que a maioria das mulheres apresentou incontinência leve. $\mathrm{O}$ antecedente de partos fórcipes associou-se à incontinência fecal.

DESCRITORES - Incontinência fecal. Menopausa. Pós-menopausa. Prevalência.

\section{INTRODUÇÃO}

A incontinência fecal (IF) é geralmente definida como perda involuntária de fezes sólidas e líquidas ${ }^{(8)}$, enquanto o termo incontinência anal inclui a perda involuntária de flatos, associada ou não a perda de fezes ${ }^{(22)}$. A incontinência fecal pode ser causa de grande desconforto, constrangimento e perda de autoconfiança, podendo interferir negativamente na qualidade de vida ${ }^{(27)}$.

Estudos mostraram que mulheres apresentam maior risco para incontinência fecal ${ }^{(19)}$. Estudo epidemiológico realizado na Nova Zelândia com pessoas acima de 65 anos, observou alta prevalência tanto em homens, como em mulheres ${ }^{(5)}$. No entanto, as mulheres parecem ser mais susceptíveis ${ }^{(12,17)}$, considerando-se como fatores de risco as lesões do nervo pudendo ou do esfíncter anal por trauma obstétrico ${ }^{(19)}$. Dentre outras causas importantes de incontinência fecal estão a lesão cirúrgica do esfíncter anal, distopias genitais e a neuropatia diabética ${ }^{(16)}$.

Estimativas da freqüência de incontinência fecal dependem do delineamento do estudo, critérios diagnósticos e seleção dos sujeitos ${ }^{(19)}$. A prevalência varia de 3\% a 28\%

\footnotetext{
${ }^{1}$ Departamento de Tocoginecologia e ${ }^{2}$ Departamento de Cirurgia da Universidade Estadual de Campinas, Campinas, SP.

Endereço para correspondência: Dr. Aarão Mendes Pinto-Neto - Departamento de Ginecologia e Obstetrícia, Universidade Estadual de Campinas - Rua Alexander Fleming, 101

- Cidade Universitária “Zeferino Vaz" - 13083-970 - Campinas, SP. E-mail: aarao@unicamp.br
} 
na população acima de 65 anos, podendo alcançar até 54,5\% em idosos sob cuidados domiciliares ${ }^{(19)}$. Em estudo populacional realizado nos Estados Unidos observou-se prevalência de 2,2\% ${ }^{(17)}$, enquanto no Reino Unido foi de $1,4 \%$ em adultos acima de 40 anos $^{(21)}$. GIEBEL et al. ${ }^{(12)}$ reportaram prevalência de $4,8 \%$ de incontinência a fezes sólidas em 500 voluntários da população geral na Alemanha. Além disso, os pacientes freqüentemente não admitem os sintomas devido ao constrangimento social ${ }^{(8)}$. FALTIN et al. ${ }^{(11)}$, estudando mulheres freqüentadoras de um serviço de saúde, observaram que apenas $20 \%$ das mulheres referiam espontaneamente sintomas de incontinência.

Existem poucos estudos avaliando especificamente mulheres na pós-menopausa, período em que a incontinência fecal passa a manifestar-se com progressiva piora.

Considerando-se a necessidade de uma abordagem integral à mulher na pós-menopausa, realizou-se este estudo, que teve como objetivos: avaliar a prevalência de incontinência fecal, estudar a intensidade dos sintomas associados e identificar os possíveis fatores a ela relacionados.

\section{PACIENTES E MÉTODOS}

Entre março de 2003 e janeiro de 2004, realizou-se estudo de corte transversal em que participaram 100 mulheres freqüentadoras do Ambulatório de Menopausa da Universidade Estadual de Campinas (UNICAMP), Campinas, SP. Foram incluídas mulheres na pós-menopausa (mínimo de 12 meses de amenorréia) ${ }^{(3)}$ e idade superior a 45 anos. Foram excluídas as mulheres com antecedente pessoal de câncer colorretal ou ginecológico, retocolite ulcerativa, doença de Crohn ou menopausa precoce (abaixo dos 40 anos de idade).

Dentre as variáveis independentes foram avaliadas características sociodemográficas e clínicas: idade atual, idade à menopausa, uso de terapia hormonal (TH) (mulheres que fizeram uso por pelo menos 1 mês ao longo da vida), tempo TH, cor, estado marital, classe socioeconômica, categorizada em A, B, C, D e $\mathrm{E}^{(2)}$, antecedentes obstétricos (gestações, abortos, cesáreas, partos fórcipes, normais e domiciliares) e diabetes mellitus. Foram avaliadas também as queixas de perda urinária, antecedentes de histerectomia, perineoplastia, cirurgia para incontinência urinária e cirurgia perianal (hemorroidectomia, fissurectomia e fistulectomia). As participantes responderam a um questionário pré-testado e estruturado para o estudo. Em seguida, realizouse exame físico para avaliação de distopias genitais (cistocele, retocele, rotura perineal) e doenças perianais (hemorróidas, fissuras, fístulas). Cistocele e retocele foram classificadas em ausentes ou graus I, II e III, respectivamente, e rotura perineal em ausente ou graus I, II, III e IV ${ }^{(25)}$.

\section{Avaliação da incontinência}

Considerou-se IF a presença de um ou mais episódios de perda de fezes sólidas ou líquidas. Não foram consideradas incontinentes as mulheres cujos sintomas não ocorreram nos últimos 30 dias. A intensidade dos sintomas associados à incontinência fecal foi avaliada através do escore de St. Mark ${ }^{(28)}$, aplicado por meio de entrevista. Este escore considera a presença e freqüência dos seguintes sintomas: incontinência a fezes sólidas, líquidas e a flatos, alteração na qualidade de vida, escape fecal, impossibilidade de inibir a defecação por 15 minutos ou menos e a necessidade de medicações constipantes. O escore varia de 0 a 24 . $\mathrm{O}$ escore 0 representa continência perfeita, enquanto o 24 refere-se à completa incontinência ${ }^{(28)}$.

\section{Análise estatística}

Utilizou-se para análise dos dados o programa SAS, versão $8.2^{(23)}$. Inicialmente todas as variáveis foram estudadas de maneira descritiva, através do cálculo de freqüências absolutas e relativas e, no caso das variáveis contínuas, através do cálculo da média, desvio-padrão, mediana, valores mínimo e máximo. Calculouse a prevalência de incontinência fecal. A seguir calculou-se o escore de St. Mark para avaliar a intensidade dos sintomas a ela associados, que foi categorizado segundo o tercil, classificandose a intensidade dos sintomas em: leve $(\leq 8)$, moderada $(9-16)$ e grave $(>16)$.

Em seguida foi estudada a associação entre a variável dependente e seus possíveis determinantes através de análise bivariada, utilizando-se como medida de associação a razão de prevalência (RP). Para tanto, as variáveis idade à menopausa e tempo de TH foram categorizadas segundo a mediana. A classe socioeconômica foi agrupada em A, B e C, D e E, a cor em branca e não-branca, o estado marital em com e sem companheiro e o uso de TH em usuárias e não-usuárias. Cistocele, retocele e rotura perineal foram agrupadas em ausente/grau I e graus II/III, respectivamente. Os antecedentes reprodutivos foram categorizados em: gestação e cesárea $(0-1, \geq 2)$, partos fórcipes e domiciliares e abortamento $(0, \geq 1)$ e partos normais $(0,1-2, \geq 3)$. Para verificar a associação entre paridade e a intensidade de incontinência fecal, foram considerados conjuntamente os partos domiciliares e normais (partos vaginais não-instrumentais), que foram categorizados em: $2 \mathrm{e} \geq 3$. As demais variáveis foram dicotomizadas em sim ou não, considerando sua presença ou ausência. Aplicou-se o teste exato de Fisher para estudar a relação entre paridade e a gravidade da incontinência fecal. O nível de significância foi fixado em $P<0,05$. Posteriormente, realizou-se análise multivariada, que consistiu no ajuste de cada variável independente às razões de prevalência da variável dependente, através do modelo de regressão de Breslow-Cox ${ }^{(26)}$. O intervalo de confiança foi fixado a 95\% (IC 95\%).

Este estudo foi aprovado pela Comissão de Pesquisa do Departamento de Tocoginecologia e pelo Comitê de Ética em Pesquisa da Faculdade de Ciências Médicas da UNICAMP. Todas as participantes assinaram o Termo de Consentimento Livre e Esclarecido.

\section{RESULTADOS}

\section{Caracterização da amostra}

A média de idade das participantes foi de 58,9 $\pm 5,9$ anos e de idade à menopausa de 47,5 $\pm 5,4$ anos. Estavam ou estiveram em uso de TH 89\%, com média de uso de 65,3 \pm 46,6 meses. Em média o número de gestações foi de $4,4 \pm 3,0$, de abortos $0,7 \pm 1,1$ e de partos normais 3,0 $\pm 2,9$ (Tabela 1 ). Das 100 mulheres, $54 \%$ eram brancas, $66 \%$ viviam com companheiro, $50 \%$ pertenciam 
às classes socioeconômicas $\mathrm{B}$ ou $\mathrm{C}$ e $50 \%$ às classes $\mathrm{D}$ ou $\mathrm{E}$. Treze por cento das participantes referiram diabetes.

Em relação às cirurgias ginecológicas, $25 \%$ eram histerectomizadas, $29 \%$ realizaram cirurgia para correção de incontinência urinária e $24 \%$ perineoplastia. Oito por cento das mulheres relataram antecedente de cirurgia perianal. Das 100 participantes, $43 \%$ referiram incontinência urinária. Ao exame físico, observou-se cistocele graus II ou III em $18 \%$ das mulheres, retocele graus II ou III em 11\%, rotura perineal graus II ou III em $28 \%$ (nenhuma apresentou rotura grau IV), hemorróida em $35 \%$ e fissura anal em $9 \%$.

Prevalência e intensidade da incontinência fecal

A prevalência de incontinência a fezes sólidas foi de $2 \%$, a fezes líquidas $15 \%$ e a flatos $27 \%$. A prevalência de incontinência fecal foi de $15 \%$ (incontinência a fezes líquidas e/ou sólidas). A maioria das participantes $(60 \%)$ apresentou incontinência fecal leve (Tabela 2).

\section{Fatores associados à incontinência fecal}

$\mathrm{Na}$ análise bivariada, a idade, o estado marital e a classe socioeconômica não se associaram à incontinência fecal. O tempo de $\mathrm{TH}$ e a idade à menopausa, bem como os antecedentes de diabetes mellitus, histerectomia e perineoplastia, também não se associaram à incontinência fecal. $\mathrm{O}$ antecedente de cirurgia para correção de incontinência urinária associou-se significativamente à incontinência fecal (RP: 4,90; IC 95\%: 1,67-14,33). Quando avaliados os antecedentes reprodutivos, apenas o antecedente de parto fórcipe associou-se significativamente à incontinência fecal (RP: 5,38; IC 95\%: 1,95-14,82). Nenhuma variável do exame anogenital associou-se à ela (Tabela 3 ).

Não houve associação entre paridade (partos vaginais nãoinstrumentais) e a intensidade da incontinência fecal (Tabela 4).

\begin{tabular}{lcccc} 
TABELA 1 - Caracterização das participantes $(\mathrm{n}=100)$ & \\
\hline Variáveis & Média & Desvio-padrão & Mínimo & Máximo \\
\hline Idade (anos) & 58,9 & 5,9 & 46 & 76 \\
Idade à menopausa (anos) & 47,5 & 5,4 & 40 & 58 \\
$\begin{array}{l}\text { Tempo de terapia hormonal } \\
\text { (meses) }\end{array}$ & 65,3 & 46,6 & 2 & 180 \\
Gestações & 4,4 & 3,0 & & \\
Abortos & 0,7 & 1,1 & 0 & 14 \\
Cesáreas & 0,6 & 0,9 & 0 & 8 \\
Partos normais & 3,0 & 2,9 & 0 & 3 \\
Partos fórcipes & 0,2 & 0,5 & 0 & 14 \\
Partos domiciliares & 1,4 & 2,7 & 0 & 3 \\
\hline
\end{tabular}

TABELA 2 - Escore de St Mark para mulheres com incontinência fecal $(n=15)$

\begin{tabular}{lll}
\hline Escore & $\mathbf{n}$ & $\mathbf{\%}$ \\
\hline Leve $(\leq 8)$ & 9 & 60,0 \\
Moderada $(9-16)$ & 5 & 33,3 \\
Grave $(>16)$ & 1 & 6,7 \\
\hline
\end{tabular}

Após análise multivariada, o antecedente de parto fórcipe (RP: 7,80; IC 95\%: 2,38-25,55) associou-se à IF (Tabela 5).

TABELA 3 - Variáveis significativamente associadas à incontinência fecal. Análise bivariada $(\mathrm{n}=15)$

\begin{tabular}{ccccc}
\hline & Variáveis & $\%$ & $\mathbf{R P}^{*}$ & IC 95\% $^{*}$ \\
\hline Incontinência fecal & Partos fórcipes & & & \\
& 0 & 53,3 & 1,00 & - \\
& $\geq 1$ & 46,7 & 5,38 & $1,95-14,82$ \\
& Cirurgia para IU & & & \\
Não & 33,3 & 1,00 & - \\
& Sim & 66,7 & 4,90 & $1,67-14,33$ \\
\hline
\end{tabular}

"RP = razão de prevalêncio

政

†IU = incontinência urinária

TABELA 4 - Relação entre a intensidade da incontinência fecal e paridade (partos vaginais não instrumentais) em mulheres na pósmenopausa $(\mathrm{n}=15)$

\begin{tabular}{lcccc}
\hline \multirow{2}{*}{ Paridade } & \multicolumn{5}{c}{ Incontinência fecal } \\
\cline { 2 - 5 } & Leve & Moderada & Grave & Total \\
\hline 2 & 2 & 2 & 0 & 4 \\
$\geq 3$ & 7 & 3 & 1 & 11 \\
Total & 9 & 5 & 1 & 15 \\
\hline
\end{tabular}

TABELA 5 - Variável significativamente associada à incontinência fecal. Análise multivariada $(\mathrm{n}=15)$

\begin{tabular}{lccc}
\hline & Variáveis & $\mathbf{R P}$ ajustada $^{*}$ & IC 95\% $^{*}$ \\
\hline Incontinência fecal & Partos fórcipes & & \\
& 0 & 1,00 & - \\
& $\geq 1$ & 7,80 & $2,38-25,55$ \\
\hline
\end{tabular}

*RP $=$ razão de prevalência ajustada por todas as variáveis

\#IC $=95 \%$ intervalo de confiança de $95 \%$

\section{DISCUSSÃO}

Este estudo teve como objetivos avaliar a prevalência de incontinência fecal e identificar seus possíveis fatores associados, numa população de mulheres na pós-menopausa. A prevalência de incontinência fecal foi de $15 \%$. Após análise multivariada, $\mathrm{o}$ antecedente de partos fórcipes associou-se à IF. Estudou-se também a intensidade dos sintomas de incontinência de acordo com o escore de St. Mark, sendo que a maioria das mulheres $(60 \%)$ apresentou escore menor ou igual a 8 pontos.

A prevalência de IF mostra resultados conflitantes na literatura, com estimativas que vão de 1,4\% em adultos com mais de 40 anos, até $62 \%$ em idosos sob cuidados domiciliares ${ }^{(19)}$. A ausência de estudos na literatura focando exclusivamente mulheres na pósmenopausa torna difícil a comparação de resultados. Estudos realizados apenas com mulheres mostraram prevalência entre $6,9 \%$ e 16,9\%, sendo as maiores freqüências encontradas naquelas em período pós-parto e em idosas ${ }^{(19)}$. A alta prevalência de mulheres incontinentes encontrada no presente estudo pode ser explicada 
pelas diferenças socioculturais entre a população avaliada e a de outros países e também porque houve uma busca ativa por meio de questionário elaborado com a finalidade de identificar mulheres com IF. Possivelmente se não se questionasse esse aspecto, a prevalência de IF teria sido menor. Além disso, trata-se de população freqüentadora de serviço de saúde especializado, onde a presença de enfermidades é mais comum.

Os escores de continência auxiliam na avaliação da gravidade da IF e seu impacto na qualidade de vida, além de serem um instrumento para análise da resposta ao tratamento clínico ou cirúrgico $^{(4)}$. Estudo prospectivo enfocando antigos escores, levou à criação do escore de St. Mark ${ }^{(28)}$ que tem mostrado alta validade clínica, principalmente no que se refere à avaliação da resposta terapêutica. A categorização do escore em tercis realizada neste estudo, visou melhor análise dos resultados. $\mathrm{O}$ fato de a maioria das mulheres ter apresentado até 8 pontos no escore pode indicar que ainda se encontram numa fase inicial da doença, com possibilidade de piora com o passar dos anos. Daí a importância do diagnóstico da IF nesta fase, para seu encaminhamento ao tratamento especializado antes do agravamento dos sintomas. Novos estudos com a utilização de questionários para avaliação da qualidade de vida são necessários, uma vez que mesmo a incontinência a flatos, considerada de menor gravidade do que a perda fecal, pode constituir grande problema para as mulheres acometidas ${ }^{(22)}$.

A idade cronológica atual e à menopausa não se associaram à IF. Isto pode ser explicado devido a média etária das mulheres estudadas ser de 58,9 anos, com poucas mulheres acima de 65 anos (apenas duas), quando a associação dessas condições patológicas com o envelhecimento é mais comum ${ }^{(11,14)}$. $\mathrm{O}$ fato de não se tratar de população predominantemente idosa também pode explicar a não associação encontrada neste estudo entre o diabetes e a IF. Em idosos, o diabetes causa neuropatia autonômica que pode levar à $\mathrm{IF}^{(24)}$.

Não se observou associação entre cor ou raça e IF. Essa relação tem sido pouco avaliada na literatura atualmente, mas um estudo epidemiológico realizado nos Estados Unidos ${ }^{(17)}$ não mostrou tal associação. A grande miscigenação racial, em especial no Brasil, torna difícil a interpretação desse dado.

Os antecedentes de uso e tempo de TH não se associaram à IF. Tal associação é pouco avaliada na literatura. Estudo realizado na América Latina ${ }^{(7)}$ apontou a menopausa como possível causa de IF em $11,2 \%$ da amostra estudada. Estudo de observação realizado na Irlanda ${ }^{(9)}$ apontou possível benefício do uso da TH em pacientes incontinentes, embora sem significância estatística. Estudos prospectivos são necessários para maiores conclusões.

$\mathrm{O}$ antecedente de cirurgia para correção de incontinência urinária associou-se à IF. As mulheres a ela submetidas provavelmente apresentavam incontinência mais grave do que aquelas que apenas referiram perda urinária, o que poderia explicar a associação entre o antecedente de cirurgia e IF. A prevalência de IF em pacientes com disfunções urinárias é reportada como sendo maior que na população geral, e 10\% a 40\% das mulheres com perda involuntária de flatos também referiram incontinência urinária e/ou prolapso pélvico $^{(20)}$. JACKSON et al. ${ }^{(13)}$ encontraram $21 \%$ de IF em mulheres com incontinência urinária e distopias genitais. Estudo realizado apenas com multíparas mostrou prevalência de $8,4 \%$ de incontinência dupla (fecal e urinária) ${ }^{(22)}$. Em estudo realizado com idosos sob cuidados domiciliares, observou-se como maior fator de risco para IF a presença de incontinência urinária ${ }^{(18)}$. Embora as distopias genitais sejam associadas na literatura à $\mathrm{IF}^{(24)}$, no presente estudo não foi encontrada tal correlação.

$\mathrm{O}$ antecedente de partos fórcipes associou-se à IF, enquanto a paridade não se associou à ela e nem à intensidade da incontinência. A associação entre parto vaginal instrumental e IF é descrita na literatura $^{(6)}$, e deve-se a danos estruturais ao esfíncter anal e/ou lesão do nervo pudendo durante o parto ${ }^{(15)}$. Estudo canadense avaliando mulheres 3 meses após o parto, apontou risco relativo após parto fórcipe de 1,4 para incontinência a fezes ou a flatos ${ }^{(10)}$. Embora existam relatos de que múltiplos partos se associem a danos cumulativos ao nervo pudendo e a maior risco de $\mathrm{IF}^{(1)}$, alguns estudos epidemiológicos não têm encontrado tal correlação, assim como no presente estudo ${ }^{(6,22)}$. Nesta série não se observou associação entre partos vaginais não-instrumentais e a intensidade da IF. Possível explicação para esse dado relacionase ao fato de que na amostra de mulheres incontinentes todas referiram dois ou mais partos, impossibilitando a comparação com mulheres de menor paridade. Essa consideração deve ser feita quando da interpretação desse resultado. Deve-se ressaltar a importância da correta assistência obstétrica durante o parto, já que a incontinência parece estar mais relacionada aos partos vaginais operatórios e com grandes lesões esfincterianas do que de fato à via de parto vaginal.

Os resultados deste trabalho devem ser interpretados considerando-se algumas limitações. Dentre elas cita-se a avaliação de população hospitalar, o uso de um escore não validado nacionalmente e o delineamento do estudo. O estudo de corte transversal possibilitou identificar algumas variáveis associadas à IF, no entanto, não se pode estabelecer inferências causais. Destaca-se que este estudo foi o primeiro a avaliar tais condições patológicas em mulheres brasileiras na pós-menopausa, e foi motivado pelo caráter multidisciplinar do atendimento que se realiza nesta instituição. Avaliações prospectivas que incluam amostras maiores poderão avaliar a real influência do estado menopausal sobre a continência e determinar as possíveis causas de IF, bem como suas repercussões sobre a qualidade de vida.

\section{CONCLUSÕES}

A prevalência de IF em mulheres na pós-menopausa foi elevada. Os resultados sugerem que a maioria das participantes apresentou incontinência leve. $\mathrm{O}$ antecedente de partos fórcipes associou-se à incontinência fecal. 
Oliveira SCM, Pinto-Neto AM, Conde DM, Góes JRN, Santos-Sá D, Costa-Paiva L. Fecal incontinence in postmenopausal women: prevalence, severity and associated factors. Arq Gastroenterol 2006;43(2):102-6.

ABSTRACT - Background - Fecal incontinence occurs more frequently in the female population and it becomes more prevalent with increasing age. There are few studies that have assessed the prevalence and severity of postmenopausal women. Aims - To investigate fecal incontinence. Patients and Methods - A cross-sectional study was performed on 100 postmenopausal women over the age of 45. Sociodemographic and clinical characteristics were evaluated, and a descriptive analysis of these characteristics was carried out. The prevalence of fecal incontinence was estimated. St. Mark's incontinence score was applied to study the severity of symptoms associated with fecal incontinence. The score was then categorized according to the tertile and symptom severity was classified as mild, moderate or severe incontinence. Bivariate and multivariate analyses were used to study the association between fecal incontinence and its likely determinants, employing the prevalence ratio. Confidence interval was set at 95\%. Results - The mean age of the patients was $58.9 \pm 5.9$ years (range, $46-76$ years). The prevalence rate was $15 \%$ for fecal incontinence. Of incontinent patients, $60 \%$ had mild incontinence. After multivariate analysis, factors associated with fecal incontinence was history of forceps delivery (prevalence ratio: 7.80; 95\% confidence interval:2.38-25.55). Conclusions - The prevalence of fecal incontinence was high in postmenopausal women. Data suggest that most women presented mild fecal incontinence. The history of forceps delivery was associated with fecal incontinence.

HEADINGS - Fecal incontinence. Menopause. Postmenopause. Prevalence.

\section{REFERÊNCIAS BIBLIOGRÁFICAS}

1. Allen RE, Hosker GL, Smith ARB, Warrell DW. Pelvic floor damage and childbirth: a neuropsychological study. Br J Obstet Gynaecol. 1990;97:770-9.

2. Almeida PM, Wickerhauser H. O critério ABA/ABIPEME - Em busca de uma atualização. São Paulo: Associação Brasileira dos Institutos de Pesquisa de Mercado; 1991.

3. Brambilla DJ, McKinklay SM, Johannes CB. Defining the perimenopause for applications in epidemiological investigations. Am J Epidemiol. 1994;140:1091-5.

4. Buchanan GN, Nicholls T, Solanki D, Kamm MA. Investigation of faecal incontinence. Hosp Med. 2001;62:533-7.

5. Campbell AJ, Reiken J, Mc Cosh L. Incontinence in the elderly: prevalence and prognosis. Age Ageing. 1985;14:65-70.

6. Chiarelli P, Murphy B, Cockburn J. Fecal incontinence after high-risk delivery. Obstet Gynecol. 2003;102:1299-305.

7. Curi LA, Genoud MT. Causas más frecuentes de incontinencia fecal en nuestro medio. Acta Gastroenterol Latinoam. 2000;30:165-8.

8. De Lillo AR, Rose S. Functional bowel disorders in the geriatric patient: constipation, fecal impaction, and fecal incontinence. Am J Gastroenterol. 2000;95:901-5.

9. Donnelly V, O'Connell PR, O'Herlihy C. The influence of oestrogen replacement on faecal incontinence in postmenopausal women. Br J Obstet Gynaecol. 1997;104:311-5.

10. Eason E, Labrecque M, Marcoux S, Mondor M. Anal incontinence after childbirth CMAJ 2002; 166:326-30.

11. Faltin DL, Sangalli MR, Curtin F, Morabia A, Weil A. Prevalence of anal incontinence and other anorectal symptoms in women. Int Urogynecol J. 2001;12:117-21.

12. Giebel GD, Lefering R, Tridl H, Blochi H. Prevalence of fecal incontinence: what can be expected? Int J Colorect Dis. 1998;13:73-7.

13. Jackson SL, Weber AM, Hull TL, Mitchinson AR, Walters MD. Fecal incontinence in women with urinary incontinence and pelvic organ prolapse. Obstet Gynecol 1997;89:423-7.

14. Kok ALM, Voohorst FJ, Burger CW, van Houten P, Kenemans P, Jansens J. Urinary and faecal incontinence in community-residing elderly women. Age Ageing. 1992;21:211-5
15. Lamah M, Kumar D. Fecal incontinence. Dig Dis Sci. 1999;44:2488-99.

16. Madoff RD, Williams JG, Caushaj PF. Fecal incontinence. N Engl J Med. 1992;4:1002-7.

17. Nelson R, Norton N, Cautley E, Furner S. Community-based prevalence of anal incontinence. JAMA. 1995;274:559-61.

18. Nelson RL, Furner S, Jesudason V. Fecal incontinence in Wisconsin nursing homes. Dis Colon Rectum. 1998;41:1226-9.

19. Nelson RL. Epidemiology of fecal incontinence. Gastroenterology. 2004;126:S3-S7.

20. Ng SC, Chen YC, Lin LY, Chen GD. Anorectal dysfunction in women with urinary incontinence or lower urinary tract symptoms. Int J Gynaecol Obstet. 2002;77:139-45.

21. Perry S, Shaw C, McGrother C, Matthews RJ, Assassa RP, Dallosso H, Williams K, Brittain KR, Azam U, Clarke M, Jagger C, Mayne C, Castleden CM; Leicestershire MRC Incontinence Study Team. Prevalence of fecal incontinence in adults aged 40 years or more living in the community. Gut. 2002;50:480-4.

22. Rizk DE, Hassan MY, Shaheen H, Cherian JV, Micallef R, Dunn E. The prevalence and determinants of health care-seeking behavior for fecal incontinence in multiparous United Arab Emirates females. Dis Colon Rectum. 2001;44:1850-6.

23. SAS [computer program]. Version 8.2. Cary: SAS Institute; 1999.

24. Schiller LR. Constipation and fecal incontinence in the elderly. Gastroenterol Clin N Am. 2001;30:497-515.

25. Shull BL. Clinical evaluation of women with pelvic support defects. Clin Obstet Gynecol. 1993;36:939-51.

26. Skov T, Deddens J, Petersen, MR, Endahl L. Prevalence proportion ratios: estimation and hypothesis testing. Int J Epidemiol. 1998;27:91-5.

27. Teunissen TAM, Lagro-Janssen ALM, van den Bosch WJHM, van den Hoogen HJM Prevalence of urinary, fecal and double incontinence in the elderly living at home. Int Urogynecol J. 2004;15:10-3.

28. Vaizey CJ, Carapeti E, Cahill JA, Kamm MA. Prospective comparison of faecal incontinence grading systems. Gut 1999;44:77-80. 